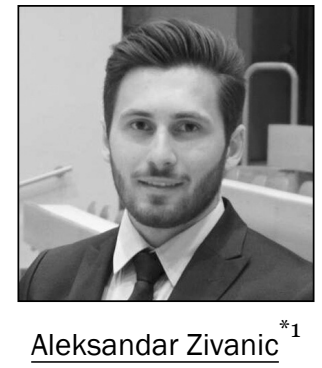

Wissenschaftlicher Mitarbeiter

Lehrstuhl für Strafrecht, Strafprozessrecht, Strafrechtsvergleichung,

Medizinstrafrecht und Rechtstheorie

Universität Konstanz

\title{
Zum kollisionsrechtlichen Umgang mit sachenrechtlichen Eigentumsvermutungen aus dem Besitz
}

\section{Einführung}

In nahezu jeder Rechtsordnung stellt der Besitz an einer (beweglichen) Sache eine vorteilhafte Rechtsposition für seinen Inhaber dar. ${ }^{{ }_{2}}$ Beispielhaft seien etwa Besitzschutzansprüche (vgl. für Deutschland die $\S \S 859$ ff. BGB; für die Schweiz die Artt. 926 ff. ZGB; für Estland die §§ 40 ff. Law of Property Act) genannt, die dem Besitzer einer beweglichen Sache zustehen. ${ }^{*}{ }^{3}$ Die Liste lässt sich freilich fortführen: So kann der bloße Besitz an einer Sache - jedenfalls in der deutschen Rechtsordnung - Gegenstand eines Kondiktionsanspruchs sein. Er stellt ein „erlangtes Etwas“ im Sinne von § 812 I 1 BGB dar. ${ }^{*} 4$ Ferner kann der Besitz deliktische Ansprüche, etwa solche aus § 823 I BGB oder § 823 II BGB in Verbindung mit § 858 BGB, auslösen. Der berechtigte, unmittelbare Besitz wird nämlich als „sonstiges Recht“ im Sinne von § 823 I BGB, ${ }^{{ }_{5}}$ die Vorschrift des § 858 BGB als „Schutzgesetz“ im Sinne von § 823 II BGB qualifiziert. ${ }^{* 6}$

Ein weiteres - besonders bedeutsames - Privileg, das der Besitz an einer beweglichen Sache mit sich bringt, ist die sogenannte Eigentumsvermutung. Eigentumsvermutungen aus dem Besitz finden sich in der deutschen Rechtsordnung einerseits in § 1006 BGB sowie andererseits in § 1362 BGB als speziellere Vorschrift für das Familienrecht. ${ }^{* 7}$ Die Vorschrift des $§ 1006$ I, II, III BGB vermutet - ihrem Wortlaut

1 Der Autor ist Wissenschaftlicher Mitarbeiter am Lehrstuhl für Strafrecht, Strafprozessrecht, Strafrechtsvergleichung, Medizinstrafrecht und Rechtstheorie bei Prof. Dr. Liane Wörner, LL.M. (UW-Madison) an der Universität Konstanz.

2 Vgl. Wendehorst, in: Münchener Kommentar zum Bürgerlichen Gesetzbuch, 8. Auflage 2021, Band 13: Internationales Privatrecht, München 2020, Art. 43 EGBGB Rn. 68 (im Folgenden: Wendehorst, in: Münchener Kommentar zum BGB).

3 Vgl. Wendehorst, in: Münchener Kommentar zum BGB, Art. 43 EGBGB Rn. 68.

4 So schon RGZ 129, 307, 311; vgl. statt vieler und mit weiteren Nachweisen Schwab, in: Münchener Kommentar zum Bürgerlichen Gesetzbuch, 8. Aufl. 2020, Band 7: Schuldrecht Besonderer Teil IV, München 2020, § 812 BGB Rn. 6.

5 Vgl. nur Wagner, in: Münchener Kommentar zum Bürgerlichen Gesetzbuch, 8. Aufl. 2020, Band 7: Schuldrecht Besonderer Teil IV, München 2020, § 823 BGB Rn. 324 mit zahlreichen weiteren Nachweisen (im Folgenden: Wagner, in: Münchener Kommentar zum BGB).

6 Vgl. BGHZ 20, 169, 171; Wagner, in: Münchener Kommentar zum BGB, § 823 BGB Rn. 595.

7 Vgl. zum Verhältnis zwischen § 1006 BGB und § 1362 BGB nur Budzikiewicz, in: Jauernig, Kommentar zum Bürgerlichen Gesetzbuch, 18. Auflage 2021, München 2021, § 1362 Rn. 1 (im Folgenden: Budzikiewicz, in: Jauernig, Kommentar zum 
nach - zugunsten des aktuellen bzw. ehemaligen (auch mittelbaren, vgl. § 868 BGB) Besitzers einer beweglichen Sache, dass er der Eigentümer der beweglichen Sache ist bzw. während seiner Besitzzeit der Eigentümer der beweglichen Sache war. Demgegenüber vermutet die Vorschrift des § 1362 I 1 BGB - ihrem Wortlaut nach - zugunsten der Gläubiger eines der Ehegatten, dass die im Besitz eines oder beider Ehegatten befindlichen beweglichen Sachen dem schuldenden Ehegatten gehören. Beide Vorschriften entfalten ihre Wirkung erst im Prozess, ${ }^{* 8}$ indem sie nach ganz überwiegender Auffassung zu einer Beweislastumkehr führen. ${ }^{*} 9$ Die hiesige Untersuchung wird sich - aus Platzgründen - lediglich auf den kollisionsrechtlichen Umgang mit der sachenrechtlichen Eigentumsvermutung, sprich derjenigen aus $§ 1006$ BGB, beschränken.

Die hier angestellten Überlegungen dürften sich allerdings auch auf das estnische Recht übertragen: Dem estnischen Zivilgesetzgeber diente das deutsche Sachenrecht als Vorbild. Sowohl im deutschen als auch im estnischen Zivilrecht gilt das Trennungs- und Abstraktionsprinzip. Hinzu kommt, dass auch dem estnischen Recht eine ähnlich formulierte sachenrechtliche Eigentumsvermutungsvorschrift bekannt ist (vgl. § 90 Law of Property Act). Letztlich ähneln sich die deutschen und estnischen Kollisionsnormen des Internationalen Sachenrechts (vgl. einerseits Artt. 43 ff. EGBGB sowie andererseits $§ § 18$ ff. Private International Law Act).

\section{Fallbeispiel: LG Darmstadt BeckRS 2016, 130834 / OLG Frankfurt am Main NJW-RR 2018, 803}

Zur Veranschaulichung der Problematik soll ein - leicht abgewandelter und verkürzter - Original-Fall, entschieden vom LG Darmstadt ${ }^{* 10}$ im Jahre 2016 sowie - im Berufungsverfahren - vom OLG Frankfurt am Main ${ }^{* 11}$ im Jahre 2018, dienen:

Bei der Klägerin (K), einem Unternehmen mit Sitz in Berlin (Deutschland), bestellte im Jahre 2010 eine Person (A) verschiedene Möbelstücke. Die Zahlung sollte dabei mittels Kreditkarte erfolgen. Die angegebenen Kreditkarten wurden zunächst vom Kreditkartenunternehmen akzeptiert. A beauftragte sodann die Beklagte (B), ein Transportunternehmen mit einer Niederlassung in Darmstadt (Deutschland), mit dem Transport der Möbel von Berlin (Deutschland) nach London (England) per Luftfracht. Zu diesem Zweck übergab K der B die Möbel. Hinterher reklamierte das Kreditkartenunternehmen die Belastungen der Kreditkarten, weil diese nicht gedeckt seien. Deshalb wurden die Möbel von der B zunächst nicht an die angegebene Adresse ausgeliefert. Im Anschluss daran forderte K von B Herausgabe der Möbelstücke. B teilte K - im Jahr 2010 - allerdings mit, dass sich die Möbelstücke nunmehr im Lager der B in Memphis (Tennessee, USA) befinden. Schließlich informierte B die K im Jahre 2013, dass die Möbelstücke nicht mehr auffindbar seien. Deshalb verlangte K von B vor dem LG Darmstadt bzw. OLG Frankfurt am Main Schadensersatz aus § 823 I BGB - wegen Eigentumsverletzung ${ }^{*}{ }^{12}$ - in Höhe von 10.00o Euro.

BGB), wonach § 1006 BGB gilt, sofern der nichtschuldende Ehegatte bereits vor der Ehe Besitzer war, vgl. zu letzterem BGH NJW 1993, 935, 936. Siehe aber auch § 1568b II BGB und dazu Ebbing, in: Erman Kommentar zum Bürgerlichen Gesetzbuch, 16. Auflage 2020, Köln 2020, § 1006 Rn. 4.

8 Keinesfalls aber nur im Zivilprozess. Die Vorschrift des § 1006 BGB findet ferner im Strafprozess und Verwaltungsprozess Anwendung, vgl. hierzu allgemein Raff, in: Münchener Kommentar zum Bürgerlichen Gesetzbuch, 8. Auflage 2020, Band 8: Sachenrecht, München 2020, § 1006 BGB Rn. 32 (im Folgenden: Raff, in: Münchener Kommentar zum BGB) (Verwaltungsprozess). Siehe ferner und aktuell BGH BeckRS 2019, 27820 (Strafprozess).

9 Vgl. zu § 1006 BGB: Spohnheimer, in: beck-online (Großkommentar, Hrsg. von Gsell/Krüger/Lorenz/Reymann), Stand: 1.11.2020, § 1006 Rn. 60 (im Folgenden: Spohnheimer, in: beck-online [Großkommentar]); Werner, Grundprobleme des $\S 1006$ - Juristische Arbeitsblätter (JA) 1983, 617, 620 und 622; siehe aber auch Raff, in: Münchener Kommentar zum BGB, $\S 1006$ BGB Rn. 66ff., wonach es sich zumindest bei § 1006 BGB aus praktischer Sicht um eine bloße Beweiswürdigungsregel handelt (so schon Heck, Grundriss des Sachenrechts, 2. Auflage 1930, Heidelberg 1930, § 33 Rn. 5); vgl. zu § 1362 BGB: Budzikiewicz, in: Jauernig, Kommentar zum BGB, § 1362 Rn. 1.

10 Vgl. LG Darmstadt, BeckRS 2016, 130834.

11 Vgl. OLG Frankfurt am Main NJW-RR 2018, 803.

12 Eine Klage aus § 823 I BGB wegen Besitzverletzung hätte von vornherein keine Aussicht auf Erfolg gehabt, weil nur der unmittelbare (vgl. § 854 I BGB), nicht aber der mittelbare (vgl. § 868 BGB) berechtige Besitz als „sonstiges Recht“ im Sinne von § 823 I BGB qualifiziert wird, vgl. hierzu nur Förster, in: Beck’scher Online-Kommentar zum BGB, Stand: 01. 11. 2020 (56. Aufl.), § 823 Rn. 156f. sowie bereits Fn 5. 
Bejaht man - wie auch das LG Darmstadt bzw. OLG Frankfurt am Main - die internationale Zuständigkeit deutscher Gerichte sowie die Geltung deutschen Sachrechts für den Hauptanspruch (vgl. Art. 4 II Rom II-VO), stellt sich im Rahmen des Hauptanspruchs (hier: § 823 I BGB) ${ }^{* 13}$ die selbstständig kollisionsrechtlich anzuknüpfende Vorfrage, ${ }^{*}{ }^{14} \mathrm{ob} \mathrm{K}$ - im Zeitpunkt der schädigenden Handlung (hier: fahrlässiger Verlust der Möbelstücke) - Eigentümerin der Möbelstücke war.

\section{Das Sachenrechtsstatut (Art. 43 I, II EGBGB): lex rei sitae}

Das deutsche Internationale Sachenrecht folgt der - nahezu weltweit anerkannten ${ }^{* 15}$ - Situs-Regel. ${ }^{* 16}$ Grundsätzlich - und vorbehaltlich der Sondernormen (vgl. Artt. 45, 46 EGBGB) - liefert die Rechtsordnung des Belegenheitsortes der Sache (sogenannte lex rei sitae) eine Antwort darauf, ob (dingliche) Rechte an einer Sache begründet wurden bzw. bestehen (vgl. Art. 43 I EGBGB). Gelangt die streitgegenständliche Sache - wie bei beweglichen Sachen nicht unüblich - in einen anderen Staat, kommt es zu einem Statutenwechsel. ${ }^{*} 7$ Für diese Fälle regelt Art. 43 II EGBGB, dass „im Ursprungsstaat entstandene Rechte an einer Sache nicht im Widerspruch zur neuen lex rei sitae ausgeübt werden können." ${ }^{*} 18$ Im Ergebnis läuft die Deutung von Art. 43 I, II EGBGB auf das Folgende hinaus: Es bedarf der Trennung von Rechtsbestands- und Rechtswirkungsstatut. ${ }^{* 19}$ Während über den Bestand einer bestimmten dinglichen Rechtslage, die noch im Ausgangsstaat begründet wurde, weiterhin allein die alte lex rei sitae entscheidet (Rechtsbestandsstatut), entscheidet die neue lex rei sitae über die Wirkung, die dieser dinglichen Rechtslage in der neuen rechtlichen Umwelt zukommt (Rechtswirkungsstatut). ${ }^{{ }^{20}}$

Im zugrundeliegenden Fall haben A und B das Eigentum an den Möbelstücken nicht erlangt - und zwar weder in Deutschland noch im Ausland. Für einen Eigentumserwerb des A in Deutschland gem. den $\S \S 929$ ff. BGB mangelte es bereits am Besitzerwerb, ${ }^{*}{ }^{21}$ wohingegen B das Eigentum mangels dinglicher Einigung nicht erlangen konnte. ${ }^{* 2}$ Damit blieb K zunächst die Eigentümerin der Möbelstücke. Mit dem Transport der Möbelstücke in die USA (Memphis, Tennessee) kam es zu einem Statutenwechsel. ${ }^{* 23}$ Die Rechte an der Sache richten sich ab diesem Zeitpunkt nach der Rechtsordnung des Bundesstaats Tennessee (vgl. Art. 43 I EGBGB in Verbindung mit Art. 4 III 1 EGBGB), wobei gem. Art. 43 II EGBGB „wohlerworbene Rechte“ - grundsätzlich - auch von der neuen Belegenheitsrechtsordnung anerkannt werden. Spätestens im Jahr 2013 waren die Möbelstücke nicht mehr auffindbar. Nach gerichtlicher Feststellung konnte die Handlung,

13 Vgl. Wandt, Gesetzliche Schuldverhältnisse, 10. Auflage, München 2020, §16 Rn. 1ff. zu den Tatbestandsvoraussetzungen für einen Anspruch aus § 823 I BGB: (1) Rechtsgutverletzung, (2) Verletzungshandlung, (3) Haftungsbegründende Kausalität, (4) Rechtswidrigkeit, (5) Verschulden, (6) Schaden sowie (7) Haftungsausfüllende Kausalität.

14 Vgl. hierzu Bernitt, Die Anknüpfung von Vorfragen im europäischen Kollisionsrecht, 2010, Tübingen, S. 147ff., insbesondere S. 157: „Die einzig überzeugende Lösung für die Anknüpfung der Vorfrage nach dem Eigentum besteht somit in einer selbstständigen Anknüpfung nach dem Kollisionsrecht der lex fori.“ - DOI: https://doi.org/10.1628/978-3-16-151422-7.

15 Vgl. hierzu Mansel, in: Staudinger Kommentar zum Bürgerlichen Gesetzbuch, 2015, Berlin 2015, Art. 43-46 EGBGB (Internationales Sachenrecht), Art. 43 Rn. 90ff. (im Folgenden: Mansel, in: Staudinger Kommentar zum BGB).

16 Vgl. zu dieser statt vieler Wendehorst, in: Münchener Kommentar zum BGB, Art. 43 EGBGB Rn. 3f. mit zahlreichen Nachweisen.

17 Vgl. hierzu allgemein Junker, Internationales Privatrecht, 3. Auflage 2019, München 2019, § 17 Rn. 7 sowie 44ff. (im Folgenden: Junker, Internationales Privatrecht). - DOI: https://doi.org/10.17104/9783406730733; Köhler, Examinatorium Internationales Privatrecht, 2. Auflage 2020, Baden-Baden 2020, Rn. 341 (im Folgenden: Köhler, Examinatorium Internationales Privatrecht). - DOI: https://doi.org/10.5771/9783845296128.

18 Wendehorst, in: Münchener Kommentar zum BGB, Art. 43 EGBGB Rn. 5.

19 Vgl. Wendehorst, in: Münchener Kommentar zum BGB, Art. 43 EGBGB Rn. 6 mit weiteren Nachweisen; weitgehend zustimmend Prütting, in: beck-online (Grosskommentar, Hrsg. von Gsell/Krüger/Lorenz/Reymann), Stand: 01.02.2020, Art. 43 Rn. 120f.

20 Vgl. umfassend Wendehorst, in: Münchener Kommentar zum BGB, Art. 43 EGBGB Rn. 6 sowie Rn. 127ff., insbesondere Rn. 133ff.

21 Das LG Darmstadt (BeckRS 2016, 130834 Rn. 26) verneinte bereits eine dingliche Einigung.

22 Vgl. zur Notwendigkeit einer dinglichen Einigung sowie eines Besitzerwerbs für einen Eigentumserwerb gem. den $\S \S 929 \mathrm{ff}$. BGB statt vieler Wieling/Finkenauer, Sachenrecht, 6. Auflage 2020, Berlin 2020. § 1 Rn. 19ff. und 23. - DOI: https://doi. org/10.1007/978-3-662-61798-4.

23 Vgl. hierzu allgemein Junker, Internationales Privatrecht, § 17 Rn. 7 sowie 44ff.; Köhler, Examinatorium Internationales Privatrecht, Rn. 341. 
die zur Unauffindbarkeit der Möbelstücke führte, lediglich in den USA (Memphis, Tennessee) begangen worden sein. Das OLG Frankfurt am Main begründete die Eigentümerstellung der K sodann mit der Vorschrift des § 1006 II BGB, indem es ausführte:

„Die [K] kann sich insofern auf § 1006 II BGB berufen, wonach zugunsten des früheren Besitzers einer beweglichen Sache vermutet wird, dass er während der Dauer seines Besitzes Eigentümer der Sache gewesen ist. Die Eigentumsvermutung zugunsten des früheren Besitzers wirkt dabei auch über die Beendigung des Besitzes hinaus so lange fort, bis sie widerlegt wird.“*24

\section{Die Bewertung der These des OLG Frankfurt am Main bei hypothetischem Inlandssachverhalt}

Um die These des OLG Frankfurt am Main einer kritischen Überprüfung zu unterwerfen, empfiehlt es sich zunächst das Fallbeispiel zu einem reinen Inlandssachverhalt abzuändern:

B wird von A beauftragt, die Möbelstücke im Inland (Deutschland) auszuliefern. Im Inland (Deutschland) verschwinden die Möbelstücke sodann im inländischen Lager der B.

Bei einem solchen, reinen Inlandssachverhalt stößt die These des OLG Frankfurt am Main auf keine Bedenken: Zunächst hatte K den Eigenbesitz (vgl. § 872 BGB) an den Möbelstücken. Gemäß $\S 1006$ I 1 BGB wurde zu diesem Zeitpunkt zugunsten der K vermutet, sie habe mit dem Eigenbesitzerwerb zugleich das Eigentum erworben - deshalb firmiert die Vorschrift des §1006 I 1 BGB auch verbreitet als Eigentumserwerbsvermutung. ${ }^{* 25}$ Indem K der B die Möbelstücke zum Zwecke des Transports übergab (und gerade nicht, um der B das Eigentum an den Möbelstücken zu übertragen), verlor sie ihren unmittelbaren Eigenbesitz. K blieb auch nicht mittelbare Besitzerin gemäß $\S 868$ BGB, weil zwischen K und B kein Besitzmittlungsverhältnis bestand, ${ }^{*}{ }^{26}$ so dass die Vermutung des $§ 1006$ I 1 BGB auch nicht über die Vorschrift des § 1006 III BGB weiterhin für sie (K) stritt. Indes streitet die Eigentumserwerbsvermutung wegen der Vorschrift des $§ 1006$ II BGB so lange für K, bis es nachweislich zu einem Eigentumsverlust gekommen ist. $^{*} 27$ Oder anders gewendet: Solange kein anderer Akteur den Eigenbesitz an den Möbelstücken erlangt, wird weiterhin vermutet, K sei Eigentümerin der Möbelstücke - deshalb firmiert die Vorschrift des §1006 II BGB auch verbreitet als Rechtsfortdauervermutung. ${ }^{* 28}$ Aus dem festgestellten Sachverhalt geht nicht hervor, dass B (oder eine dritte Person) den Eigenbesitz (vgl. § 872 BGB) an den Möbelstücken erlangt hat. Deshalb wird gemäß $§ 1006$ II BGB zugunsten der K (weiterhin) vermutet, sie sei im Zeitpunkt der schädigenden Handlung (Verlust der Möbelstücke) Eigentümerin der Möbelstücke gewesen.

Widmet man sich nun wieder dem Ausgangsfall, der einen grenzüberschreitenden Bezug aufweist, stößt die Annahme, die deutsche Eigentumsvermutung - scil. § 1006 II BGB - streite zugunsten der K, doch auf einige Bedenken, denn:

\footnotetext{
OLG Frankfurt am Main NJW-RR 2018, 803, 804 Rn. 26.

Vgl. statt vieler Marco Brand, Der Organbesitz, 2015, Tübingen, S. 200 mit zahlreichen Nachweisen in Fn 10.

26 Erforderlich hierfür wäre nämlich ein konkretes Besitzmittlungsverhältnis (vgl. hierzu allgemein Vieweg/Werner, Sachenrecht, 8. Auflage 2018, München 2018, § 2 Rn. 29 und 32) zwischen K und B, das deshalb nicht bestand, weil zwischen K und B kein schuldrechtlicher Vertrag zustande kam (vgl. hierzu LG Darmstadt BeckRS 2016, 130834 Rn. 21) sowie kein gesetzliches Besitzmittlungsverhältnis bestand.

27 Vgl. hierzu statt vieler Thole, in: Staudinger Kommentar zum BGB, 2019, Berlin 2019, § 1006 Rn. 53 mit zahlreichen Nachweisen (im Folgenden: Thole, in: Staudinger Kommentar zum BGB).

28 Siehe etwa Fritzsche, in: beck-online Kommentar, Stand: 01.11.2020 (56. Aufl.), § 1006 Rn. 9.
} 


\section{Zur Rechtsprechung des Bundesgerichtshofs (BGH) zum kollisionsrechtlichen Umgang mit der Eigentumsvermutung aus dem Besitz}

\subsection{Die Auffassung des VII. BGH-Zivilsenats}

Schon in der frühen Nachkriegszeit äußerte sich der VII. BGH-Zivilsenat zum kollisionsrechtlichen Umgang mit der Eigentumsvermutung aus dem Besitz. Der VII. BGH-Zivilsenat postulierte, dass allgemeine Beweislastregeln, zu denen auch die Eigentumsvermutung zugunsten des Besitzers gehöre, nicht dem Prozessstatut (sog. lex fori), sondern dem Belegenheitsstatut (lex rei sitae) unterfallen. ${ }^{* 2}$ Deshalb, so der VII. BGH-Zivilsenat, richtet sich „die Frage, ob für den Besitzer einer beweglichen Sache eine Eigentumsvermutung besteht oder nicht, nach dem Recht des Ortes, an dem die Sache während der Besitzzeit desjenigen sich befand oder befindet, der das Eigentum an ihr in Anspruch nimmt und sich auf Grund seines früheren oder gegenwärtigen Besitzes auf die Eigentumsvermutung beruft. "“*30 Die Passage des BGH-Urteils erscheint bei erster Lektüre etwas kryptisch. Gleichwohl lässt sich ihre Kernaussage bei näherer Lektüre leicht entziffern: Beansprucht eine Person das Eigentum an einer Sache und ist sie ihr gegenwärtiger Besitzer, so streitet die Eigentumsvermutung des § 1006 BGB nur für sie, sofern sich die Sache im Inland (Deutschland) befindet (vgl. Art. 43 I EGBGB). Beansprucht eine Person das Eigentum an einer Sache und ist sie ihr früherer Besitzer, so streitet die Eigentumsvermutung des § 1006 BGB nur für sie, sofern sich die Sache während ihrer Besitzzeit im Inland (Deutschland) befunden hat (vgl. Art. 43 I EGBGB).

Der Leser mag nun aufhorchen. Im Fallbeispiel befand sich die Sache (die Möbelstücke) - im Zeitpunkt der schädigenden Handlung - gerade nicht (mehr) im Inland (Deutschland), sondern im Ausland (Tennessee, USA). Nach der Rechtsprechung des VII. BGH-Zivilsenats kann zugunsten der K gerade nicht die inländische Eigentumsvermutung (§1006 BGB), sondern allenfalls die ausländische Eigentumsvermutung, nämlich diejenige der Rechtsordnung des Bundesstaates Tennessee eingreifen. Das OLG Frankfurt am Main hat insofern entgegen der Rechtsprechung des VII. BGH-Zivilsenats entschieden.

\subsection{Die Auffassung des IV. BGH-Zivilsenats}

Indes hat eine Entscheidung des IV. BGH-Zivilsenats aus dem Jahre 1994 für Verwirrung rund um den (richtigen) kollisionsrechtlichen Umgang mit der Eigentumsvermutung aus dem Besitz gesorgt - und möglicherweise auch das Urteil des OLG Frankfurt am Main beeinflusst. Der IV. BGH-Zivilsenat äußerte seinerzeit zweierlei: Einerseits greife zugunsten des gegenwärtigen Besitzers einer - nunmehr im Inland belegenen - beweglichen Sache die Eigentumsvermutung des § 1006 I 1 BGB auch dann, wenn der Eigenbesitz an der Sache im Ausland begründet wurde. ${ }^{*}{ }^{31}$ Andererseits wirke die Eigentumsvermutung aus $§ 1006$ I 1 BGB fort, wenn die Sache ins Ausland verbracht wird. ${ }^{*}{ }^{2}$ Gerade die letztere Aussage steht evident im Widerspruch zu der Auffassung des VII. BGH-Zivilsenats, der bei einem Statutenwechsel allenfalls die ausländische Eigentumsvermutung aus dem Besitz heranzieht. Indes darf die Aussage des IV. BGH-Zivilsenats nicht überschätzt, jedenfalls aber nicht kontextfrei betrachtet werden. Der IV-BGH-Zivilsenat formuliert nämlich weiter: „Deshalb kann hier [im zugrunde liegenden Fall] offen bleiben, ob die Eigentumsvermutung des Art. 930 ZGB [...] ebensoweit [reicht] wie diejenige aus § 1006 BGB und ob sie, wenn deutsches Recht nicht eingriffe, auch ihrerseits die Bekl. von weiteren Beweisen freistellen würden." ${ }^{*} 33$ Der IV. BGHZivilsenat hielt es lediglich für nicht entscheidungserheblich welche Eigentumsvermutung aus dem Besitz, sprich die deutsche oder die schweizerische, heranzuziehen ist. Er hat die Streitfrage, ob die deutsche Eigentumsvermutung bei einem Statutenwechsel fort gilt, schlicht offengelassen. ${ }^{*} 3$ Dieser Streitfrage gilt es daher auf den Grund zu gehen.

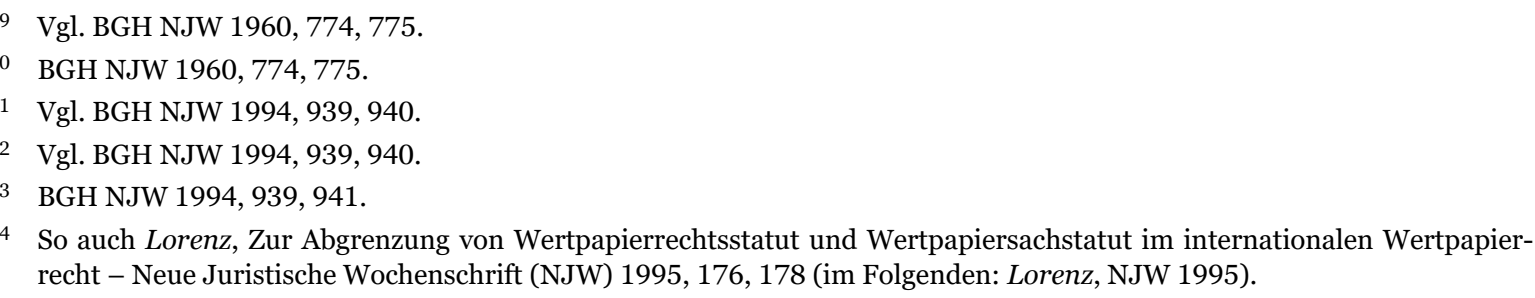


Dem Urteil des OLG Frankfurt am Main ist insofern nur zuzustimmen, wenn (1) die Eigentumsvermutung aus dem Besitz gemäß der lex fori kollisionsrechtlich anzuknüpfen, (2) die Eigentumsvermutung aus dem Besitz als wohlerworbenes Recht im Sinne von Art. 43 II EGBGB zu begreifen oder (3) eine wesentlich engere Verbindung gemäß Art. 46 EGBGB anzunehmen ist.

\section{Lex fori-Anknüpfung der Eigentumsvermutung aus dem Besitz?}

Der VII. BGH-Zivilsenat verneinte eine kollisionsrechtliche Anknüpfung der Eigentumsvermutung aus dem Besitz anhand der lex fori, weil allgemeine Beweislastregeln nicht so eng mit der besonderen Ausgestaltung des Prozessverfahrens in den einzelnen Ländern zusammenhängen. ${ }^{*} 35$ Die Auffassung des BGH entspricht dem heutigen europäischen Verständnis: Der Unionsgesetzgeber unterwirft Beweislastregelungen und gesetzliche Vermutungen sowohl für vertragliche als auch für außervertragliche Schuldverhältnisse der lex causae (vgl. Art. 18 Rom I-VO; Art. 22 Rom II-VO). Dieser Umstand dürfte als hinreichendes Indiz dafür gelten, dass Beweislastregelungen rechtskreisübergreifend materiell-rechtlich qualifiziert werden. ${ }^{*}{ }^{6}$ Nach alledem scheidet eine Anknüpfung der Eigentumsvermutung aus dem Besitz anhand der lex fori aus.

\section{Die Eigentumsvermutung aus dem Besitz als wohlerworbenes Recht im Sinne von Art. 43 II EGBGB?}

Offen bleibt, ob die Eigentumsvermutung aus dem Besitz als ein „wohlerworbenes Recht“ im Sinne von Art. 43 II EGBGB begriffen werden kann. Diese Frage wurde - soweit ersichtlich - noch nicht eingehend scil. monographisch - diskutiert. ${ }^{*}{ }^{37}$ Bislang äußerten sich Armbrüster ${ }^{*} 38$, Behr $^{*} 39$, Lorenz $^{*} 40$ und Einsele ${ }^{*}{ }^{41}$ hierzu: Während Lorenz davon spricht, dass es sich bei der Eigentumsvermutung aus dem Besitz „nicht um einen abgeschlossenen Erwerbstatbestand, sondern um die inhaltliche Ausgestaltung eines Sachenrechts [handelt] "“*2, hält es Einsele für eine „doch wohl etwas merkwürdige Vorstellung “* 43 die Eigentumsvermutung aus dem Besitz als wohlerworbenes Recht im Sinne von Art. 43 II EGBGB zu bezeichnen. Auch Behr vertritt den Standpunkt, die Eigentumsvermutung aus dem Besitz könne kein wohlerworbenes Recht darstellen, weil Eigentumsvermutungen keinen „Einfluss auf die materielle Rechtslage [haben]. ${ }^{* * 4}$ Armbrüster hingegen scheint einer Qualifikation als „wohlerworbenes Recht“ nicht abgeneigt zu sein, indem er ausführt: „Die belgische Eigentumsvermutung könnte dann, verstanden als Bestandteil der sog. sachenrechtlichen Prägung der Gegenstände, nach deren Verbringung ins Bundesgebiet [Deutschland] hier fortbestehen. “*45

\footnotetext{
35 Vgl. BGH NJW 1960, 774, 775.

36 Siehe dazu auch Varga, in: beck-online (Grosskommentar, Hrsg. von Gsell/Krüger/Lorenz/Reymann), Stand: 15.02.2016, Art. 22 Rom II-VO Rn. 3 mit weiteren Nachweisen in Fn 7.

37 Vgl. auch Armbrüster, Eigentumsvermutung nach § 1006 BGB und Statutenwechsel im internationalen Privatrecht - Praxis des Internationalen Privat- und Verfahrensrechts (IPRax) 1990, 25 Fn 26 (im Folgenden: Armbrüster, IPRax 1990).

38 Vgl. Armbrüster, IPRax 1990, 25.

39 Vgl. Behr, Ausländische Inhaberaktien und § 1006 BGB: Ein Beitrag zum Anwendungsbereich gesetzlicher Eigentumsvermutungen, in: Festgabe für Otto Sandrock zum 65. Geburtstag, 1995, Frankfurt am Main, S. 159, 164ff. (im Folgenden: Behr, in: Festschrift für Otto Sandrock zum 65. Geburtstag).

40 Vgl. Lorenz, NJW 1995, 176, 177 f.

41 Vgl. Einsele, Kollisionsrechtliche Behandlung von Wertpapieren und Reichweite der Eigentumsvermutung des §1006 BGB Praxis des Internationalen Privat- und Verfahrensrechts (IPRax) 1995, 163, 164 (im Folgenden: Einsele, IPRax 1995).

42 Lorenz, NJW 1995, 176, 178.

43 Einsele, IPRax 1995, 163, 164.

44 Behr, in: Festschrift für Otto Sandrock zum 65. Geburtstag, S. 159, 165.

45 Armbrüster, IPRax 1990, 25.
} 


\subsection{Auslegung des Art. 43 II EGBGB und die Wurzeln der Eigentumsvermutung nach überwiegender Auffassung in Rechtsprechung und Lehre}

Aufschluss darüber, welcher Auffassung der Vorzug gebührt, kann nur eine Auslegung des Art. 43 II EGBGB liefern. Mittlerweile, so das überwiegende Schrifttum ${ }^{*} 46$, dem sich nunmehr auch der BGH ${ }^{*} 47$ angeschlossen hat, bewirkt Art. 43 II EGBGB eine temporale Spaltung des Sachstatuts. Hierbei gilt es - wie bereits gezeigt wurde - zwischen Rechtsbestands- und Rechtswirkungsstatut zu unterscheiden. Legt man ein solches Verständnis Art. 43 II EGBGB zugrunde, bedeutet dies für die Eigentumsvermutung aus dem Besitz, dass sie - weil es sich bei ihr um eine „Wirkung eines dinglichen Rechts handelt“*48 - dem Rechtswirkungsstatut unterfällt und damit wandelbar ist. Konkret: Es findet die Eigentumsvermutung aus dem Besitz der Belegenheitsrechtsordnung Anwendung ${ }^{*} 49$ - und das Urteil des OLG Frankfurt am Main hielte rechtlicher Nachprüfung nicht stand.

Wirft man einen näheren Blick auf das heutige Verständnis der Eigentumsvermutung aus dem Besitz (§ 1006 I 1 BGB), stößt die kollisionsrechtliche Beurteilung derselben anhand des Rechtswirkungsstatuts auf Bedenken. Wie bereits ausgeführt, vermutet § 1006 I 1 BGB, dass der Besitzer mit dem Erwerb seines Eigenbesitzes (vgl. § 872 BGB) auch das Eigentum erworben hat. ${ }^{*} 0$ Überwiegend wird vertreten, die Vorschrift des § 1006 BGB fuße auf dem Traditionsprinzip. ${ }^{*}{ }^{51}$ Vor diesem Hintergrund vertritt ein Teil der Lehre die Auffassung, die deutsche Eigentumsvermutung finde in grenzüberschreitenden Bezügen immer und nur dann Anwendung, wenn der Besitzerwerb im Inland (Deutschland) stattgefunden hat. ${ }^{* 2}$ Spohnheimer formuliert hierzu: „Folgt nun ein Land, in dem der Besitzer den Besitz ergriffen hat, für die Übereignung dem Konsensualprinzip, so erscheint es fernliegend, ihn an der Eigentumsvermutung des § 1006 [BGB] partizipieren zu lassen. ."*53 Die Eigentumserwerbsvermutung gilt - dieser Auffassung zufolge - als ein wohlerworbenes Recht. ${ }^{*} 4$ Sie unterliegt mithin dem Rechtsbestandsstatut. Dies entspricht im Ergebnis der kontextgelösten Formulierung des IV. BGH-Zivilsenats. Für das hiesige Fallbeispiel würde dies bedeuten, dass für K, weil sie den Eigenbesitz an den Möbelstücken in Deutschland erlangt hat, weiterhin die deutsche Eigentumserwerbsvermutung aus § 1006 BGB streitet - und das Urteil des OLG Frankfurt am Main hielte rechtlicher Prüfung stand.

\subsection{Qualifikation der Vorschrift des § 1006 BGB als Ausfluss des Traditionsprinzips?}

Indes ist die Auffassung von Rechtsprechung und herrschender Lehre, die Vorschrift des § 1006 BGB sei Ausfluss des Traditionsprinzips, zweifelhaft. Die in der Literatur schon früh geäußerte Kritik zu dieser These, ist nahezu verstummt: Während Rosenberg ${ }^{*} 55$ die Eigentumsvermutung - getreu ihrem Wortlaut - schon immer als Rechtszustandsvermutung begriff, erhob Armbrüster ${ }^{*}{ }^{56}$ den weitergehenden Einwand, auch Rechtsordnungen, die nicht dem Traditionsprinzip folgen, kennen ähnliche Eigentumsvermutungsregelungen aus dem Besitz; schon deshalb könne § 1006 BGB seine Wurzeln nicht im Traditionsprinzip

\footnotetext{
46 Siehe bereits Fn 19.

47 Vgl. BGH NJW-RR 2010, 983, 984.

48 So die Bezeichnung bei Wendehorst, in: Münchener Kommentar zum BGB, Art. 43 EGBGB Rn. 6 sowie Rn. 127ff., insbesondere Rn. 133ff.; so auch Einsele, IPRax 1995, 163, 164.

49 So ausdrücklich Behr, in: Festschrift für Otto Sandrock zum 65. Geburtstag, S. 159, 166.

50 Siehe dazu Marco Brand, Der Organbesitz, 2015, Tübingen, S. 200; Berger, in: Jauernig Kommentar zum BGB, 18. Auflage 2021, München 2021, § 1006 Rn. 1.

51 Vgl. Spohnheimer, in: beck-online (Großkommentar, Hrsg. von Gsell/Krüger/Lorenz/Reymann), Stand: 1.11.2020, § 1006 Rn. 6 mit weiteren Nachweisen.

52 Vgl. Spohnheimer, in: beck-online (Großkommentar, Hrsg. von Gsell/Krüger/Lorenz/Reymann), Stand: 1.11.2020, § 1006 Rn. 68f.; Thole, in: Staudinger Kommentar zum BGB, § 1006 Rn. 105.

53 Spohnheimer, in: beck-online (Großkommentar, Hrsg. von Gsell/Krüger/Lorenz/Reymann), Stand: 1.11.2020, § 1006 Rn. 68.

54 Auf diese Konsequenz hinweisend Armbrüster, IPRax 1990, 25.

55 Vgl. Rosenberg, Beweislast, 5. Auflage 1965, München, Berlin 1965, § 16 (S. 226ff.).

56 Vgl. Armbrüster, IPRax 1990, 25.
} 
haben. Hierzu lässt sich folgendes ergänzen: Selbst Rechtsordnungen, die - wie die schweizerische Rechtsordnung - gleichermaßen das Traditionsprinzip zugrunde legen, verstehen die Eigentumsvermutung aus dem Besitz (vgl. Artt. 930, 931 ZGB) nicht als Ausfluss des Traditionsprinzips, sondern begreifen sie als bloße Wahrscheinlichkeitsfolgerung. ${ }^{*} 57$ Wirft man einen Blick in die BGB-Materialien, so findet ebendiese Folgerung - Eigentumsvermutung als bloße Wahrscheinlichkeitsfolgerung - eine gewichtige Stütze. Aus den BGB-Materialien geht nämlich nicht eindeutig hervor, dass es sich bei § 1006 BGB um eine Vorschrift handelt, die auf das Traditionsprinzip zurückzuführen ist. Der historische BGB-Gesetzgeber begründete die Aufnahme der Vorschrift nämlich unter anderem mit den Worten: „Diese Vermuthung entspreche [...] der Anschauung des deutschen Rechtes [und] dem Code 2279 “* ${ }^{58}$ Platziert man die subjektiv-historische Auslegungsmethode in einer Rangfolge der Auslegungsregeln zu Recht - für sie streitet sowohl die Gesetzesbindung gem. Art. 20 III, 97 I GG als auch das Gewaltenteilungs- und Demokratieprinzip ${ }^{*} 59$ - an die oberste Stelle, findet die Auffassung der Rechtsprechung und herrschenden Lehre methodisch keine Stütze, weil sich der historische BGB-Gesetzgeber an Art. 2279 Code Civil orientierte, dem französischen Zivilrecht das Traditionsprinzip aber fremd ist.

Für die kollisionsrechtliche Behandlung von Eigentumsvermutungen aus dem Besitz - scil. § 1006 BGB - bedeutet dies, dass solche dem Rechtswirkungsstatut unterfallen. Eigentumsvermutungen aus dem Besitz erweisen sich nicht als sachenrechtliche Prägung der Gegenstände. Vielmehr entscheidet die aktuelle lex rei sitae darüber, ob und inwiefern der Besitz an einer Sache eine Eigentumsvermutung zugunsten des Besitzers statuiert. Das OLG Frankfurt am Main hätte insofern, weil sich die Möbelstücke in Tennessee (USA) befanden, die ausländische Eigentumsvermutung aus dem Besitz - sofern die Rechtsordnung des Bundestaates Tennessee (USA) eine solche kennt - heranziehen müssen. Indes hätte eine solche K wohl kaum geholfen, ihre Eigentümerstellung zu beweisen, zumal sie zu diesem Zeitpunkt weder unmittelbare noch mittelbare Besitzerin der Möbelstücke war. K hatte, indem sie die Möbelstücke aus der Hand gab, „die Lebenserfahrung gegen sich und damit die Obliegenheit der Beweissicherung." “60 Die Heranziehung deutschen Rechts - hier: § 1006 II BGB - konnte nach alledem, insbesondere unter Zugrundelegung des Art. 43 I, II EGBGB, in jedem Fall nicht überzeugen.

\section{Wesentlich engere Verbindung gemäß Art. 46 EGBGB?}

Damit ist aber nicht, jedenfalls nicht abschließend festgestellt, dass die deutsche Eigentumsvermutung aus $\S 1006$ II BGB nicht auf anderem Wege Eingang in das Verfahren hätte finden können. Auch das Internationale Sachenrecht strebt danach, das sachnächste Recht zu ermitteln. ${ }^{* 61}$ Dies belegt schon die Kodifizierung des Art. 46 EGBGB. ${ }^{* 2}$ Die Vorschrift des Art. 46 EGBGB statuiert, dass das Recht derjenigen Rechtsordnung anzuwenden ist, mit der eine wesentlich engere Verbindung besteht. An dieser Stelle besteht Einigkeit darüber, dass Art. 46 EGBGB eine restriktiv zu handhabende Ausnahmenorm darstellt. ${ }^{* 63}$ Mansel $^{* 64}$ fordert für die Einschlägigkeit des Art. 46 EGBGB die Ermittlung aller Sachverhaltsumstände und der relevanten Interessen, wobei ebendiese Gesamtabwägung immer auf einen konkreten Anknüpfungsgegenstand

57 Vgl. Winkler, Die Rechtsvermutungen aus dem Besitz, 1969, Zürich, S. 24.

58 Vgl. Prot. S. 4052 (Mugdan, Materialien zum Bürgerlichen Gesetzbuch, Bd. III, S. 520; siehe aber auch Armbrüster, IPRax 1990, 25, 26 Fn 15 unter Verweis auf eine Mindermeinung in Prot. S. 3372 (Mugdan, Materialien zum Bürgerlichen Gesetzbuch, Bd. III, S. 519): „Immerhin habe die Vermuthung zu Gunsten des Eigenbesitzers eine gewisse statistische Wahrscheinlichkeit für sich, daß der Eigenbesitzer Eigenthümer sei.“

59 Vgl. hierzu kürzlich Höpfner/Schneck, Verfassungsrechtliche Vorgaben für die Wahl der Rechtsanwendungsmethode - AD Legendum 2020, 201, 202ff.; Rüthers, Die Entwicklung der juristischen Methodenlehre nach dem Zweiten Weltkrieg Anmerkungen und Hypothesen - AD Legendum 2020, 217, 219f. jeweils mit weiteren Nachweisen.

60 Armbrüster, IPRax 1990, 25, 26.

61 Vgl. allgemein zu dieser Funktion des IPR („Prinzip der engsten Verbindung“) Junker, Internationales Privatrecht, § 5 Rn. 5ff.; speziell für das Internationale Sachenrecht Mansel, in: Staudinger Kommentar zum BGB, Art. 43 Rn. 16.

62 Es handelt sich bei der Vorschrift um eine sogenannte Ausweichklausel, vgl. Mansel, in: Staudinger Kommentar zum BGB, Art. 46 Rn. 5.

63 Vgl. statt vieler Spickhoff, in: beck-online Kommentar zum BGB, Stand: 01.11 .2020 (56. Aufl.), Art. 46 Rn. 1: „Notbehelf“; deutlicher Wendehorst, in: Münchener Kommentar zum BGB, Art. 46 EGBGB Rn. 2: „extreme Ausnahmefälle“.

64 Mansel, in: Staudinger Kommentar zum BGB, Art. 46 Rn. $33 f$. 
bezogen sein muss. Unter Anknüpfungsgegenstand ist dabei - so Wendehorst ${ }^{*} 65$ - die konkrete Rechtsfrage im Sinne des Begehrens des Anspruchstellers (hier: Schadensersatz aus Delikt, genauer: wegen Eigentumsverletzung) zu verstehen.

Legt man all dies dem Fallbeispiel zugrunde, kommt man zu dem Ergebnis, dass die deutsche Eigentumsvermutung aus § 1006 II BGB über Art. 46 EGBGB Anwendung findet: Hierfür sprechen schon die objektiven Umstände des konkreten Einzelfalls. ${ }^{* 66}$ Einerseits haben die Parteien (K und B) eine gemeinsame Niederlassung in Deutschland, andererseits fand auch die Übergabe der Möbelstücke in Deutschland statt. ${ }^{* 67}$ Hinzu kommt, dass auch der Hauptanspruch (hier: Schadensersatz aus Delikt) deutschem Sachrecht unterliegt - und zwar aus ähnlichen Erwägungen: Die Parteien haben im Inland (Deutschland) ihren gewöhnlichen Niederlassungsort (vgl. Art. 4 II Rom II-VO). Darüber hinaus spielte sich auch der sonstige Sachverhalt - etwa die Beauftragung der B durch A, der Vertragsschluss zwischen A und K - im Inland (Deutschland) ab. Abschließend gelangten die Möbelstücke - und insofern für A und K nicht ersichtlich - unter außergewöhnlichen Umständen in die USA (Tennessee). A hatte B nämlich lediglich damit beauftragt, die Möbel nach England zu transportieren. Nur aufgrund der Tatsache, dass das Kreditkartenunternehmen die Kreditkartenzahlung nicht akzeptierte, gelangten die Möbel in die USA. Nimmt man das Prinzip der Ermittlung des sachnächsten Rechts ernst, kann dem Statutenwechsel im konkreten Fall keine Bedeutung beigemessen werden. Damit bleibt es im Ergebnis bei der Feststellung des OLG Frankfurt am Main: Zugunsten der K streitet die deutsche Eigentumsvermutung aus § 1006 II BGB.

\section{Fazit}

Der kollisionsrechtliche Umgang mit der sachenrechtlichen Eigentumsvermutung aus dem Besitz erfolgt in Rechtsprechung und Lehre nicht immer einheitlich. Die Ursache ist freilich schnell ausgemacht: Während der VII. BGH-Zivilsenat die Eigentumsvermutung aus dem Besitz dem Rechtswirkungsstatut unterwarf, erlaubte ein Urteil des IV. BGH-Zivilsenat eine gegenteilige Interpretation dergestalt, dass es sich bei der Eigentumsvermutung aus dem Besitz um ein wohlerworbenes Recht im Sinne von § 43 II EGBGB handelt, sie mithin dem Rechtsbestandsstatut unterliegt. Hinzu kommt, dass das nationale Verständnis der Eigentumsvermutung aus dem Besitz (§ 1006 BGB) als Eigentumserwerbsvermutung, die ihren Ursprung vermeintlich im Traditionsprinzip habe, für Verwirrung im kollisionsrechtlichen Umgang mit derselben sorgt. Hier konnte sowohl methodisch als auch rechtsvergleichend gezeigt werden, dass die Eigentumsvermutung aus dem Besitz keinerlei Bezüge zum Traditionsprinzip aufweist. Vielmehr handelt es sich bei ihr um eine bloße Wahrscheinlichkeitsregelung. Damit steht fest: Grundsätzlich entscheidet die Belegenheitsrechtsordnung, ob der Besitz an einer Sache eine Eigentumsvermutung für den Besitzer bewirkt (Rechtswirkungsstatut). Getreu dem Motto ,jede Regel hat eine Ausnahme“ lässt sich über Art. 46 EGBGB ein gegenteiliges Ergebnis erzielen. So können die objektiven Umstände des Einzelfalls - trotz Statutenwechsels - für die Anwendung des Rechts des ehemaligen Belegenheitsstaates streiten. Besonderes Gewicht haben hierbei vor allem der gemeinsame gewöhnliche Aufenthalt der Parteien sowie der Ort der Sachübergabe.

65 Wendehorst, in: Münchener Kommentar zum BGB, Art. 46 EGBGB Rn. 24.

66 Siehe zu diesen allgemein Mansel, in: Staudinger Kommentar zum BGB, Art. 46 Rn. 35.

67 Vgl. Mansel, in: Staudinger Kommentar zum BGB, Art. 46 Rn. 35 unter Hinweis auf den gemeinsamen gewöhnlichen Aufenthalt sowie den Ort der Sachübergabe. 